\title{
ISOLATION OF CATECHIN FROM Acacia catechu Willdenow ESTIMATION OF TOTAL FLAVONOID CONTENT IN CAMELLIA SINENSIS KUNTZE AND CAMELLIA SINENSIS KUNTZE VAR. ASSAMICA COLLECTED FROM DIFFERENT GEOGRAPHICAL REGION AND THEIR ANTIOXIDANT ACTIVITIES
}

\author{
Padam P. Acharya*, Giri R. Genwali* and Meena Rajbhandari** \\ *Central Department of Chemistry, Tribhuvan University, Kirtipur, Kathmandu, Nepal. \\ **Research Center for Applied Science and Technology, Tribhuvan University, Kathmandu, Nepal.
}

\begin{abstract}
Catechin, a flavanol was isolated from the ethylacetate soluble portion of the water extract of red heartwood of Acacia catech $u$ Willd. The isolated catechin was characterized by comparing melting point, $\mathrm{R}_{\mathrm{f}}$ values, $\mathrm{UV}$ and IR spectra with authentic catechin. Quantitative determination of flavonoids in different parts of Camellia sinensis Kuntz and Camellia sinensis Kuntz var. assamica under different extraction conditions, collected from Ilam, Jhapa and Pachthar was carried out using aluminum chloride colorimetric method. Catechin was used as the standard for the calibration of flavanoids. The greatest total flavonoid content was revealed in the methanol extract of the stem collected from Ilam (192.18 mg catechin/g sample) and the lowest was determined in 50\% methanol extract of old leaves collected from Jhapa (19.21 mg catechin/g sample). The antioxidant activity of the selected tea extracts was determined by DPPH assay. The extract having the highest flavonoid content showed the lowest $\mathrm{IC}_{50}$ demonstrating the positive correlation between radical scavenging activity $\left(\mathrm{IC}_{50}\right)$ and total flavonoid content. The flavonoids contributed $65.8 \%$ to free DPPH radical scavenging of the extracts. The result indicated that the Camellia plant is the rich source of high value polyphenol compounds as natural antioxidants to use in preventive medicines and food industry.
\end{abstract}

Kewwords: Acacia catechu; Catechin; Camellia species; Total flavonoid content; Antioxidant activity;Correlation.

\section{INTRODUCTION}

Polyphenols are the class of chemical compounds synthesized by fruits, vegetables, teas, cocoa and other plants that possess certain health benefits. They are an integral part of human diet and responsible for overall organoletpic properties of plant foods. Plant polyphenols have drawn increasing attention due to their potent antioxidant properties and their marked effects in prevention of various oxidative stress associated diseases such as cardiovascular, cancer and neurodegenerative diseases $1-4$.

Catechin, a flavanol is a polyphenolic compound found in tea, coca and several fruits. The terminal three leaves of the Camellia plant is used for the production of green, oolong and black tea. The green tea contains 30 to $50 \%$ polyphenols 5 mainly epicatechin, epicatechin gallate, epigallocatechin and epigallocatechin gallate on the dry weight basis 6,7 . They constitute $90 \%$ of the total flavonoids 8 and a cup of green tea contains about 150 to $210 \mathrm{mg}$ of polyphenols 9 . In black tea, catechins are converted to theaflavines 6,10 . Many researches have demonstrated that the phytochemicals present in tea have beneficial effect as they act as a free radical scavenger. They have shown strong antioxidant activity like vitamin $\mathrm{C}, \mathrm{E}$ and carotenoids ${ }^{11}$, anti-inflamatory ${ }^{12}$, cholesterol lowering 13 , antiviral and antibacterial activities ${ }^{14,15}$.

Although processed teas were well investigated for their polyphenol content and antioxidant activities no

Author for correspondences: Meena Rajbhandari, RECAST, T. U., Kirtipur. Email: karmacharyameena@gmail.com. 
information is available on the polyphenol content and antioxidant effect of Nepalese Camellia plants. Hence the present study was conducted to quantify the total flavonoid content in different parts and different extracts of Nepalese Camellia plants collected from different geographical regions using catechin as a standard, determination of antioxidant activity of selected extracts using DPPH free radical scavenging assay and to find out correlation between total flavonoids content and their antioxidant activity. This will help to find new sources of safe and inexpensive natural antioxidants to use them in food and pharmaceutical preparations to replace synthetic antioxidants.

\section{MATERIALS AND METHODS}

\section{Chromatographic materials and Chemicals}

TLC foils (precoated) Silica gel 60 GF254, 0.2 $\mathrm{mm}$ and TLC foils (precoated) Cellulose F, 0.2mm, were purchased from Merck, Darmstadt, Germany. Sephadex LH-20 was purchased from Pharmacia Biotech, Uppasala, Sweden. DPPH and $( \pm)$ catechin was purchased from Sigma Chemical Company, USA. Aluminum chloride was purchased from sd finechemicals. All other chemicals were of analytical grade.

\section{Plant materials}

Red heartwood of A. catechu Willd was collected from Jhapa district in January 2012. Fresh young and old leaves twigs and stems of $C$. sinensis and C. sinensis var. assamica were collected from the tea gardens of Panchthar, Ilam and Jhapa districts in February 2012. They were authenticated by Prof. R. P. Chaudhary, Central Department of Botany, Tribhuvan University, Kathmandu, Nepal. Voucher specimens were deposited at the Research Centre for Applied Science and Technology, RECAST, Tribhuvan University.

\section{Extraction of A. catechu heartwood and isolation of catechin}

The fine chips of the heartwood of A. catechu was boiled with water. The water was evaporated and the dried extract was subjected to liquid-liquid extraction using ethylacetate and water. The ethylacetate phase was dried in a rotator evaporator to get a viscous mass. The ethylacetate extract was subjected to gel column chromatography on Sephadex LH-20 using methanol. Altogether four major fractions were collected. The fractions were examined for the presence of catechin by TLC analysis in different solvent systems and the chromatogram was visualized by spraying with vanillin- $\mathrm{HCl}$ reagent which produce dark pink colour against white background, is a specific reagent for the detection of condensed tannins. The catechin accumulated fraction was further subjected to Sephadex LH-20 column chromatography using methanol and three major fractions were collected. The fraction containing catechin with minor impurities was purified by Sephadex LH-20 column chromatography using 50\% aqueous methanol. The white powder thus obtained was further purified by recrystallization with hot water.

\section{Extraction of $C$. sinensis and $C$. sinensis var assamica}

An amount of $20 \mathrm{~g}$ each of the different parts (old leaves, young leaves and stem) of dried and powdered plant samples were extracted with methanol in a soxhlet extraction apparatus. The residue was extracted with $50 \%$ aqueous methanol under reflux. Similarly, each $20 \mathrm{~g}$ of different parts of dried and powdered samples were percolated with $70 \%$ acetone and subjected to ultrasound-assisted extraction. The extracts were filtered and the solvent was evaporated in a rotary evaporator under reduced pressure.

\section{Determination of Total Flavonoid Content in different extracts}

\section{Preparation of standard}

The total flavonoid content was determined by aluminum chloride colorimetric assay (16). Various concentrations of standard catechin $(0.75 \mathrm{mg} / \mathrm{mL}, 0.5 \mathrm{mg} /$ $\mathrm{mL}, 0.25 \mathrm{mg} / \mathrm{mL}$ and $0.125 \mathrm{mg} / \mathrm{mL}$ ) were prepared. An aliquot of $1 \mathrm{~mL}$ catechin of each concentration in methanol was added to $10 \mathrm{~mL}$ volumetric flask containing $4 \mathrm{~mL}$ of double distilled water. At the zero

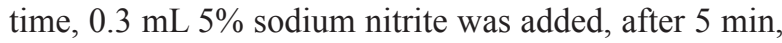
$0.3 \mathrm{~mL}$ of $10 \% \mathrm{AlCl}_{3}$ was added and at $6 \mathrm{~min}, 2 \mathrm{~mL}$ of $1 \mathrm{M}$ sodium hydroxide was added to the mixture. Immediately, the total volume of the mixture was made up to $10 \mathrm{~mL}$ by the addition of $2.4 \mathrm{~mL}$ double distilled water and mixed thoroughly. Absorbance of the pink color mixture was determined at $510 \mathrm{~nm}$ versus a blank containing all reagents except catechin. The average absorbance values obtained at different concentrations of catechin were used to plot the calibration curve.

\section{Preparation of sample}

Various concentrations of the extracts $(1 \mathrm{mg} / \mathrm{mL}, 0.5$ $\mathrm{mg} / \mathrm{mL}, 0.25 \mathrm{mg} / \mathrm{mL}$ and $0.125 \mathrm{mg} / \mathrm{mL}$ ) were prepared. Following the procedure described for standard, absorbance for each concentration of extract was recorded. Total flavonoid content of the extracts was expressed as mg catechin equivalents (CE) per gram of sample in dry weight $(\mathrm{mg} / \mathrm{g})$. Total flavonoid content is calculated by using the formula: $\mathrm{C}=\mathrm{cV} / \mathrm{m}$ where, $\mathrm{C}=$ total flavonoid content $\mathrm{mg} \mathrm{CE} / \mathrm{g}$ dry extract, $\mathrm{c}=$ concentration of catechin obtained from calibration curve in $\mathrm{mg} / \mathrm{mL}, \mathrm{V}=$ volume of extract in $\mathrm{mL}, \mathrm{m}=$ mass of extract in gram 


\section{Statistical Analysis}

All the experiments were carried out in triplicates and data reported are mean \pm standard deviation. Calculation of linear correlation coefficient and correlation analysis were carried out using MS Office Excel 2007. The linear regression equation for a straight line is, $\mathrm{Y}=$ $\mathrm{mx}+\mathrm{c}$ where, $\mathrm{Y}=$ absorbance of extract, $\mathrm{m}=$ slope of the calibration curve, $\mathrm{x}=$ concentration of extract, $\mathrm{c}=$ intercept. Using this regression equation, concentrations of extracts were calculated. From the calculated values of concentration of each extract, the total flavonoid content was calculated.

Determination of antioxidant activities using 2, 2-diphenyl-1-picrylhydrazyl free radical

Antioxidant activity of the selected extracts was assessed (17) using DPPH free radical. DPPH solution (0.1 mM) was prepared by dissolving $3.9 \mathrm{mg}$ of DPPH in $100 \mathrm{~mL}$ methanol and stirred overnight at $4^{\circ} \mathrm{C}$. Thus prepared purple colored DPPH free radical solution was stored at $-20^{\circ} \mathrm{C}$ for further use.

Three different concentrations $(5,10$ and $15 \mu \mathrm{g} / \mathrm{mL})$ of methanolic solutions of each extracts were prepared by the serial dilution of the stock solution of the respective extract. To each $0.5 \mathrm{~mL}$ extract solution, $2.5 \mathrm{~mL} 0.1$ $\mathrm{mM}$ methanolic DPPH solution was added. A control was prepared by mixing $0.5 \mathrm{~mL}$ distilled water and 2.5 $\mathrm{mL} 0.1 \mathrm{mM}$ methanolic DPPH solution. These samples were shaken well and kept in dark for $30 \mathrm{~min}$ at room temperature. The absorbance was measured at $517 \mathrm{~nm}$ against the blank solution consisting $2.5 \mathrm{ml} \mathrm{MeOH}$ and $0.5 \mathrm{~mL}$ distilled water. The radical scavenging activity was expressed as the radical scavenging percentage using the equation where; $A_{S}=$ absorbance of sample solution, $\mathrm{A}_{\mathrm{b}}=$ absorbance of blank and $\mathrm{A}_{\mathrm{C}}=$ absorbance of control

$$
\% \text { scavenging }=\left\lfloor\frac{(A s-A b)}{A c}\right\rfloor \times 100
$$

$\mathrm{IC}_{50}$ value is the concentration of sample required to scavenge $50 \%$ of DPPH free radical and was calculated from the graph of radical scavenging activity against the concentration of extracts. Statistically, the correlation between antioxidant activity and total flavonoid content, TFC was determined by plotting $\mathrm{IC}_{50}(\mu \mathrm{g} / \mathrm{mL})$ against TFC (mg/g).

\section{RESULT AND DISCUSSION}

\section{Characterization of catechin from $A$. catechu}

Repeated column chromatograph of the ethylacetate phase of the water extract of A. catechu over Sephadex LH 20 yielded catechin which was purified by recrystallization with hot water. Thin layer chromatography behavior and the melting point of the isolated catechin is in good agreement with the authentic catechin. The UV spectrum of the isolated and authentic catechin showed absorption bands at 220 and $277 \mathrm{~nm}$. The IR spectra of the catechin has a broad band around 3400-2600 $\mathrm{cm}^{-1}$ region corresponding to the aliphatic and aromatic $\mathrm{C}-\mathrm{H}$, phenolic and alcoholic $\mathrm{O}-\mathrm{H}$ stretching. Other stretching's were comparable with IR spectra of authentic catechin.

\section{Total flavonoid contents in $C$. sinensis and $C$. sinensis var. assamica}

Total flavonoid contents in the extracts were determined by reaction with sodium nitrite followed by the development of colored flavonoid-aluminum complex formation using aluminum chloride in alkaline condition which was monitored spectrophotometrically at maximum wavelength of $510 \mathrm{~nm}$. Total Flavonoid content of the extracts was calculated from the regression equation of calibration curve $\left(y=0.002 x ; R^{2}=1\right)$ and expressed as $\mathrm{mg}$ catechin equivalents (CE) per gram of sample in dry weight $(\mathrm{mg} / \mathrm{g})$.

Total flavonoid content differs according to the nature of the extract and the plant parts used. However, topography of the collection site is not significant. The total flavonoid content in methanol and 70\% acetone extracts were relatively high when compared with 50\% aqueous methanol extract in all samples collected from Ilam, Jhapa and Panchthar. Similarly, the total flavonoid present in the young leaves and the stem collected from all three geographical regions were relatively high in comparison to the old leaves. The highest amount of flavonoid was detected in the methanol extract of the stem collected from Ilam (192.18 mg catechin/g sample) and the lowest amount was detected in 50\% aqueous methanol extract of old leaves collected from Jhapa (19.21 mg catechin/g sample). The methanol and 70\% acetone extracts of young leaves collected from Ilam, Jhapa and Panchthar contain nearly the same amounts of flavonoids ranged from 116.08-140.86 mg catechin/g sample. On the other hand, the total flavonoid contents in the methanol and $70 \%$ acetone extracts of stem collected from three different regions showed little variation and ranged from 103.57-192.18 mg catechin/g sample. All the three extracts of the old leaves collected from all three regions showed the presence of relatively low amount of total flavonoids than the stem and young leaves. However, $70 \%$ acetone extract of old leaves showed the presence of somehow higher amount of flavonoid (84.82 $\mathrm{mg}$ catechin/g sample) than the methanol extract (70.30 $\mathrm{mg}$ catechin/g sample). The total flavonoid content in different parts of Camellia plant is given in Table 1 .

Tea, particularly green tea is a source of polyphenols and thus acts as potential antioxidants. Many reports are available about the total flavonoid content and the 
Table 1: Total Flavonoid Content in different parts of various Camellia plants

\begin{tabular}{|c|c|c|c|c|c|}
\hline Plants & Parts & Collection site & Methanol (mg/g) & $50 \%$ Methanol $(\mathrm{mg} / \mathrm{g})$ & $70 \%$ Acetone $(\mathrm{mg} / \mathrm{g})$ \\
\hline C. sinensis & Young leaves & Ilam & $129.4625 \pm 3.4841$ & $48.25 \pm 2.5851$ & $140.8625 \pm 3.9053$ \\
\hline \multicolumn{2}{|l|}{ C. sinensis } & Jhapa & $134.125 \pm 1.2316$ & $30.25 \pm 4.0234$ & $123.5125 \pm 2.7673$ \\
\hline \multicolumn{2}{|c|}{ C. sinensis var assamica } & Panchthar & $116.0875 \pm 2.5245$ & $45.5125 \pm 2.0532$ & $132.45 \pm 3.1635$ \\
\hline C. sinensis & Old leaves & Ilam & $70.3 \pm 4.428$ & $30.6375 \pm 2.9999$ & $84.6625 \pm 2.9093$ \\
\hline \multicolumn{2}{|l|}{ C. sinensis } & Jhapa & $62.55 \pm 4.3557$ & $19.2125 \pm 3.8721$ & $78.2125 \pm 6.4719$ \\
\hline \multicolumn{2}{|c|}{ C. sinensis var assamica } & Panchthar & $40.575 \pm 2.4273$ & $31.275 \pm 2.2354$ & $84.825 \pm 4.9073$ \\
\hline C. sinensis & Stem & Ilam & $192.187 \pm 1.4117$ & $37.55 \pm 2.5705$ & $142.612 \pm 7.9321$ \\
\hline \multicolumn{2}{|l|}{ C. sinensis } & Jhapa & $124.20 \pm 1.5953$ & $55.962 \pm 2.8960$ & $141.15 \pm 4.5401$ \\
\hline \multicolumn{2}{|c|}{ C. sinensis var assamica } & Panchthar & $103.575 \pm 0.7361$ & $48.5625 \pm 0.9092$ & $121.925 \pm 1.9917$ \\
\hline
\end{tabular}

antioxidant activity but the result varies depending on the assay method. The aluminum chloride colorimetric assay of $50 \%$ aqueous methanol extract Camellia, green tea revealed the presence of $19.17 \mathrm{mg}$ catechin/g sample 18 and water extract revealed $47 \mathrm{mg}$ epicatechin/g sample ${ }^{19}$. These values are very low when compared with our results. The total polyphenol contents and antioxidant activities depends on the geographical regions of growth, season of collection, storage condition and extraction methods.

\section{DPPH radical scavenging assay}

The DPPH assay is based on the capability of an antioxidant to donate a hydrogen radical or an electron to DPPH radical, which is stable free radical with deep violet color. When an odd electron become paired in the presence of free radical scavenger of antioxidant agent, DPPH radicals get reduced to corresponding hydrazine, DPPH-H form ${ }^{20}$ and the solution gets decolorized from its initial deep violet to light yellow color. The degree of fall in the absorbance is measured spectrophotometrically and is proportional to the concentration of the antioxidant.

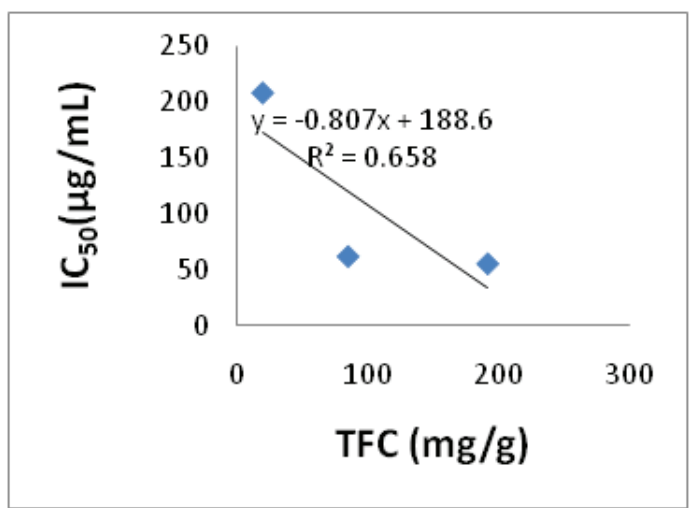

Fig 1: Correlation between radical scavenging activity and total flavonoid content.

Selected extracts, methanol extract of C.sinensis (stem/ Ilam, TFC 192.18), 50\% methanol extract of C. sinensis (old leaves/Jhapa, TFC 19.21) and 70\% acetone extract of C. sinensis var. assamica (old leaves/Panchthar, TFC 84.82) were assessed for their free radical scavenging capacities using DPPH free radicals. The absorbance values were measured at wavelength $517 \mathrm{~nm}$ for different concentration of extracts and the control. These values are used to calculate the percentage inhibitions of $\mathrm{DPPH}^{*}$ radicals against the samples. The $\mathrm{IC}_{50}$ values of various extracts were calculated from the percentage inhibitions at various concentrations and found to be $55.47,209.13$ and $62.13 \mu \mathrm{g} / \mathrm{mL}$ respectively. The extract containing high amount of flavonoids showed high radical scavenging activity.

The correlation between antioxidant activity and TFC had been determined by plotting $\mathrm{IC}_{50}(\mu \mathrm{g} / \mathrm{mL})$ against TFC (mg/g). The relationship between total flavonoid contents and free radical scavenging activity, FRSA of the samples is shown in Fig 1. A direct correlation between radical scavenging activity $\left(\mathrm{IC}_{50}\right)$ and $\mathrm{TFC}$ of the samples was demonstrated by linear regression analysis. The relationship between radical scavenging activity (Y) and TFC (X) revealed coefficient of determination $\mathrm{R}^{2}$ of 0.658 . These result suggested that flavonoid compounds contributed $65.8 \%$ to free DPPH radical scavenging of the extracts.

It is well known that green tea is a potential source of natural antioxidant and its antioxidant power is correlated to the total phenolic content. The antioxidant activity of phenolics is due to their redox properties which allow them to act as reducing agents, hydrogen donors, singlet oxygen quencher and metal chelators $21-22$. Synthetic antioxidants used in food industry such as butylatedhydroxy anisole (BHA) exhibit genotoxic and carcinogenic effect, while butylatedhydroxy toluene (BHT) is proven to cause hemorrhage23-25. Thus, the natural antioxidants from plants are of greater interest in preventive medicines and food industry 26 and their activity is considered to be multifunctional to prevent oxidation in complex food system 27. 


\section{CONCLUSION}

The results of the investigation indicated that the different parts of Camellia plants are the rich source of high value polyphenol compounds as natural antioxidants. These compounds were easily extractable with methanol and $70 \%$ acetone rather than $50 \%$ methanol. Therefore, not only the terminal three leaves of shoots of Camellia plants but the twigs and stems can also be used for preparing tea and other home herbal remedies which may have possible beneficial implications in human health such as in the treatment and prevention of cancer, cardiovascular disease and other pathologies.

\section{ACKNOWLEDGEMENT}

This work is supported by the financial support of Volkswagen Foundation, Germany and Nepal Academy of Science and Technology, Khumaltar. The author is grateful to Prof. U. Lindequist, University of Greifswald for providing authentic catechin and Sephadex and Prof. S. M. Tuladhar, RECAST, T.U. for providing DPPH.

\section{REFERENCES}

1 Rasmussen, S.E., Frederiksen, H., Struntze Krogholm, K., Poulsen, L. 2005. Dietary proanthocyanidins: Occurrence, dietary intake, bioavailability, and protection against cardiovascular disease. Molecular Nutrition and Food Research. 49: 159-174.

2 Arts, I.C., Hollman, P.C. 2005. Polyphenols and disease risk in epidemiological studies. American Journal of Clinical Nutrition. 81: 317-325.

3 Hertog, M.G., Feskens, E.J., Hollman, P.C., Katan, M.B.,Kromhout, D. 1994. Dietary flavonoids and cancer risk in the Zutpen Elderly Study. Nutrition and Cancer. 22: 175-184.

4 Cole, G.M., Lim, G.P., Yang,F., Teter, B., Begum, A., Ma, Q., Harris-White, M.E., Frautschy, S. A. 2005. Prevention of Alzheimer's disease: Omega-3 fatty acids and phenolic antioxidant interventions. Neurobiology and Aging. 26 (1): 133-136.

5 Dreosti, I. E. 2000. Antioxidant polyphenols in tea, coca and wine. Nutrition. 16: 692-694

6 Yang, G. Y., Liao, J. Li, C et. al. 2000. Effect of black and green tea polyphenols on c-jun phosphorylation and $\mathrm{H}(2) \mathrm{O}(2)$ production in transformed and non-transformed human bronchial cell lines: possible mechanisms of cell growth inhibition and apoptosis induction. Carcinogenesis. 21: 2035-39

7 Brocher, A., Keen, C. L., Hannum, S. M. et. al. 2000. Cocoa and Chocolate: Composition, bioavailability, and health implications. Journal of Medicinal Foods. 3: 77-105

8 Tijburg, L. B. M., Mattern, T., Folts, J. D., et al 1997. Tea flavonoids and cardiovascular diseases: A review. Critical Reviews in Food Science and Nutrition. 37: 771-785

9 Bravo L. 1998. Polyphenols: Chemistry, Dietary Sources, Metabolism, and Nutritional Significance. Nutrition Reviews. 56: 317-333

10 Yang, C. S., Landau, J. M. 2000. Effect of tea consumption on nutrition and health. Journal of Nutrition. 130: 2409-2412

11 Sharma,S.,StrutzmanJD,Kellof,G.J.,Steele V.E.1994.creening of potential chemo preventive agents using biochemical mark-

ers of carcinogenesis. Cancer Research. 54: 5848-5855.

12 2007. Green tea polyphenols as an anti-oxidant and antiinflammatory agent for cardiovascular protection. Drug Targets. 7 (2): 135-144

13 Koo, S. I., Noh, K. S. 2007. Green tea as inhibitor of the intestinal absorption of lipids: potential mechanism for its lipid-lowering effect. Journal of Nutritional Biochemistry. 18 (3): 179-183

14 Weber,J.M.,Ruzindana-Umunyana,A., Imbeault, L ., S i r c a r, S. 2003. Inhibition of adenovirus infection and adenain by green tea catechins. Antiviral research. 58 (2): 167-173

15 Friedman, M., Henika, P. R., Levin, C. E., Mandrell, R. E., Kozukue, N. 2006. Antimicrobial activities of tea catechins and theaflavins and tea extracts against Bacillus cereus. Journal of Food Protection. 69 (2): 354-361

16 Zhishen, J., Mengcheng, T., Jianming, W. 1999. The determination of flavonoid contents in mulberry and their scavenging effects on superoxide radicals. Food Chemistry. 64: $555-559$

17 Brand-William, Cuvelier, W., Berset, C. 1995. Use of a free radical method to evaluate antioxidant activity Lebensmittelwissenschaft und Technologie. 28 (1): 25-30

18 Hajimahamoodi, M., Hanifeh, M., Oveisis, N., Sadeghi, N., Junnat, B. 2008. Determination of total antioxidant capacity of green teas by the ferric reducing/antioxidant power assay. Irian Journal of Environment, Health, Science, and Engineering. 5: 167-172

19 Lee, K. W., Kim, y. J., Lee, H. J., Lee, C. Y. 2003. Cocoa has more phenolic phytochemicals and a higher antioxidant capacity than teas and red wine. Journal of Agriculture and Food Chemistry. 51: 7292-7295

20 Piaxao, N., Perestrelo, R., Marques, J.C., Camara, J.S. 2007. Relationship between antioxidant capacity and total phenolic content of red, rose and white wines. Food Chemistry. 105: 204-214.

21 Pietta, P. G. 2000. Flavonoids as Antioxidants. Journal of Natural Products. 63: 1035-1042

22 Canadanovic-Brunet, J. M., Djilas, S. M., Cetkovic, G. S., Thumbas, V. S. 2005. Free radical scavenging activity of wormwood (Artemisia absinthium L) extracts. Journal of Science Food and Agriculture. 85: 265-272

23 Chen C., Pearson M.A and Gray I.J. 1992. Effects of synthetic antioxidants. (BHA, BHT and PG) on the mutagenicity of IQlike compounds. Food Chemistry. 43 (3): 177-183

24 Ito N., Horose M., Fukushima H., Tsudo T., Shairai T., Tatenastu M. 1986. Studies on antioxidants: their carcinogenic and modifying effects on chemical carcinogenesis. Food Chemistry and Toxicology.24: 1071-1092

25 Kahl, R. and kappus H. 1993. Toxicity of synthetic antioxidants BHA and BHT in comparison with natural antioxidants vitamin E. Zeitschrift fur Lebensmittel-Untersuchung und-Forschung. 196 (4): 329-338

26 Staden .J. V., Moyo, M., Ndhlala, A. R. 2010. Natural Antioxidants: Fascinating or Mythical Biomolecules? Molecules. 15: 6905-6930

27 Wang T., Jonsdottir, R., Olafsdottir, G. 2009. Total phenolic compounds, radical scavenging and metal chelation of extracts from Icelandic seaweeds. Food Chemistry. 116(1):240-248 